\title{
Anemia e gravidez
}

\author{
Carlos Antonio B. Montenegro, ${ }^{1}$ Flávia C. dos Santos, ${ }^{1}$ Jorge de Rezende-Filho ${ }^{1 *}$
}

\section{Resumo}

A anemia carencial é um problema nutricional comum, e sua prevalência é alta entre mulheres em idade reprodutiva. $\mathrm{O}$ ginecologista-obstetra que atua como médico de atenção primária pode desempenhar um papel fundamental na detecção e tratamento de anemia por deficiência de ferro. Além disso, o tratamento e a profilaxia da anemia durante o período gestacional funcionam de forma a prevenir distúrbios que podem repercutir na infância, como parto prematuro, baixo peso ao nascimento e dificuldades cognitivas do recém-nascido. A prevalência de anemia na gravidez é de aproximadamente $40 \%$, mais de $50 \%$ dos quais por deficiência de ferro. As necessidades de ferro são seis vezes maiores na gravidez e, não podendo ser cobertas pela dieta, são supridas parcialmente pelas reservas maternas. Atendendo à hemodiluição fisiológica da gravidez, a anemia é definida pelo CDC como a presença de hemoglobina menor que 10,5-11 g/dL. Recomenda-se a suplementação universal na gravidez de $60 \mathrm{mg}$ por dia de ferro elementar. A anemia reduz a resistência da mulher grávida a infecções e aumenta de duas a três vezes a incidência de complicações na gravidez e no parto. Em gestantes anêmicas, a taxa de partos pré-termo é três vezes mais frequente e está acrescida em duas vezes a mortalidade perinatal. Hemorragias ante e pós-parto são mais comuns em pacientes com anemia e, frequentemente, fatais. Na Ásia, a anemia é a segunda causa de morte materna. $\mathrm{O}$ tratamento da anemia ferropriva é feito com sulfato ferroso, $200-400 \mathrm{mg}$, três vezes ao dia por via oral; excepcionalmente administra-se o ferro por via intravenosa. Quando a hemoglobina é menor que 6-7 g/dL, indica-se o concentrado de hemácias.

Descritores: Anemia; Deficiência de ferro; Hemorragia.

\section{Abstract}

\section{Anemia and pregnancy}

Iron deficiency anemia is a common nutritional problem, whose prevalence is high among women of childbearing age. Obstetrician/gynecologists, acting as primary care physicians for women, can play a pivotal role in the detection and treatment of iron deficiency anemia. Moreover, treatment and prophylaxis of anemia during the gestational period work to prevent that may impact on childhood as premature birth, low birth weight and cognitive difficulties of the newborn. The incidence of anemia in pregnancy is approximately $40 \%$, and in over $50 \%$ of the time, it is due to iron deficiency. Iron requirements are six times higher in pregnancy and, since they may not be covered by the diet alone, they are partially supplied by the maternal reserves. Given the physiological
1. Departamento de Ginecologia e Obstetrícia. Maternidade-Escola. Universidade Federal do Rio de Janeiro. Rio de Janeiro, RJ, Brasil.

\author{
*Endereço para correspondência: \\ Av. Atlântica 822/102 \\ Rio de Janeiro, RJ, Brasil. CEP: 22010-000. \\ E-mail: rezendef@terra.com.br
}

Revista HUPE, Rio de Janeiro, 2015;14(2):29-33

doi: $10.12957 /$ rhupe. 2015.18350

Recebido em 29/05/2014. Aprovado em 01/07/2014.

hemodilution in pregnancy, anemia is defined by the CDC as the presence of hemoglobin $<10.5-11 \mathrm{~g} / \mathrm{dL}$. Universal iron supplementation in pregnancy is recommended in a dose of $60 \mathrm{mg} /$ day of elemental iron. Anemia reduces resistance to infections in pregnant women and promotes a two to three fold increase in the incidence of complications in pregnancy and childbirth.In anemia, the rate of preterm births is three times higher and perinatal mortality is increased by two times. Pre-and postpartum hemorrhages are more common in anemic pregnant women, and often fatal. In Asia, anemia is the second leading cause of maternal death. The treatment of iron deficiency anemia is made with ferrous sulfate, $200-400 \mathrm{mg}$, three times daily, orally; exceptionally iron is administered intravenously. When hemoglobin is $<6-7 \mathrm{~g} / \mathrm{dL}$, Aninfusion of erythrocyte concentrate is indicated.

Keywords: Anemia; Iron deficiency; Hemorrhage.

\section{Resumen}

\section{Anemia y embarazo}

La anemia por deficiencia nutricional es un problema nutricional común y su prevalencia es alta entre las mujeres en edad reproductiva. El ginecólogo-obstetra que actúa como médico de atención primaria puede desempeñar un papel clave en la detección y tratamiento de la anemia por deficiencia de hierro. Además de eso, el tratamiento y la profilaxis de la anemia durante el período gestacional funcionan para prevenir trastornos que pueden repercutir en la infancia, como parto prematuro, bajo peso al nacer y dificultades cognitivas del recién nacido. La prevalencia de la anemia en el embarazo es de aproximadamente $40 \%$, más del $50 \%$ por deficiencia de hierro. Las necesidades de hierro son seis veces más altas en el embarazo, y no pueden ser cubiertas por la dieta, se suplen parcialmente con las reservas maternas. 
Observando la hemodilución fisiológica del embarazo, la anemia se define por el CDC como la presencia de hemoglobina $<10,5-11 \mathrm{~g} / \mathrm{dL}$. Se recomienda el suplemento universal en el embarazo de $60 \mathrm{mg}$ /día de hierro elemental. La anemia reduce la resistencia de la mujer embarazada a las infecciones y aumenta de dos a tres veces la incidencia de complicaciones en el embarazo y el parto. En las mujeres embarazadas con anemia, la tasa de partos pretérmino es tres veces más frecuente y se incrementa por el doble la mortalidad perinatal. Hemorragias ante y post-parto son más comunes en pacientes con anemia, y con frecuencia fatales. En Asia, la anemia es la segunda causa de muerte materna. El tratamiento de la anemia por deficiencia de hierro se realiza con sulfato ferroso, 200-400 mg, tres veces al día por vía oral; excepcionalmente se administra el hierro por vía intravenosa. Cuando la hemoglobina es $<6-7 \mathrm{~g} / \mathrm{dl}$, se indica el concentrado de hematíes.

Palabras clave: Anemia; Deficiencia de hierro; Hemorragia.

\section{Introdução}

A anemia nutricional, queda anormal das taxas de hemoglobina no sangue, é resultante da deficiência de ferro, vitamina B12 e ácido fólico. Outras causas de anemia incluem as doenças parasitárias como a malária e as verminoses, além das hemoglobinopatias hereditárias como a talassemia. A prevalência mundial de anemia na mulher grávida é de aproximadamente 40\%, e em mais de 50\% dos casos é por deficiência de ferro. Na Ásia, a anemia é a segunda causa de mortalidade materna, com incidência de $14 \%{ }^{1}$

\section{Caracterização dos tipos de anemia}

Há três tipos de anemia nutricional, caracterizados de acordo com o fator deficiente e responsável pela queda da hemoglobina: a anemia hipocrômica ferropriva, por deficiência de ferro; a anemia megaloblástica, por deficiência de ácido fólico; e a anemia perniciosa, pela deficiência de vitamina B12.

Em uma perspectiva global, a deficiência de ferroé a mais comum. É encontrada com alta prevalência em mulheres com idade fértil, assim como em gestantes e em puérperas. ${ }^{2} \mathrm{Em}$ alguns países em desenvolvimento, a anemia ferropriva passa a ser a regra ao invés de ser a exceção com uma prevalência de aproximadamente $52 \% .^{3}$ A anemia por deficiência de ácido fólico, embora muito frequente na gravidez em virtude das necessidades aumentadas de folato, habitualmente ocorre juntamente com a ferropriva e permanece não diagnosticada. Presume-se que em países em desenvolvimento ocorra em $20 \%$ a $25 \%$ das grávidas. A anemia por carência de vitamina B12 tem menor prevalência.

\section{Etiologia}

A elevada incidência de anemia nos países em desenvolvimento resulta dos efeitos combinados e aditivos de dieta inadequada, doenças, infestações recorrentes, multiparidade e pequeno intervalo interpartal.

As necessidades de ferro e de ácido fólico são seis vezes maiores no último trimestre da gravidez. Essas elevadas demandas de ferro não podem ser cobertas exclusivamente pela dieta, sendo supridas parcialmente pelas reservas maternas. Estudos prévios recomendavam dose profilática de suplementação de ferro um tanto altas, de 100-200 mg, diariamente. ${ }^{4}$ Considerando os potenciais efeitos colaterais da ingestão de ferro, é importante que seja definida a menor dose de ferro eficaz para cumprir as metas previstas. A suplementação de ferro pode diminuir a absorção de outros minerais como o zinco e aumentar o estresse oxidativo tanto em nível local como nos intestinos e de forma sistêmica. ${ }^{5,6} \mathrm{Um}$ estudo realizado com gestantes na Dinamarca avaliou o efeito de 20, 40, 60 e $80 \mathrm{mg}$ de ferro administrados diariamente a partir de 18 semanas até o parto. A dose de $20 \mathrm{mg}$ pareceu ser ineficaz para prevenir a deficiência de ferro em número substancial de mulheres. Contudo, $40 \mathrm{mg}$ de ferro preveniram anemia em mais de $95 \%$ das mulheres. Não houve diferença significativa no status de ferro entre mulheres que usaram 40, 60 e 80 mg de ferro. ${ }^{7}$ E, uma vez que as reservas são, em geral, repostas tão somente fora da gravidez, mulheres que têm pequenos intervalos entre os partos sofrem de anemias crônicas e progressivas. A demanda pela absorção de ferro aumenta consideravelmente durante a gravidez, de $0,8 \mathrm{mg}$ por dia nas primeiras dez semanas para 7,5 mg por dia nas últimas dez semanas. Durante todo o período gestacional, a demanda média pela absorção de ferro é de 4,4 mg por dia. $8,9,10$

Quando o nível de ferro da gestante está reduzido apreciavelmente, ela não é capaz de sintetizar a hemoglobina. À conta da hemodiluição fisiológica da gravidez, os níveis de hemoglobina que configuram a anemia são bem mais baixos que os existentes fora da gestação. Assim, a definição de anemia na gravidez recomendada pelo Centro de Controle e Prevenção de Doenças (CDC) é o valor de hemoglobina menor que $11 \mathrm{~g} / \mathrm{dL}$ no primeiro trimestre da gestação, menor 
que $10,5 \mathrm{~g} / \mathrm{dL}$ no segundo trimestre e menor que $11 \mathrm{~g} /$ dL no terceiro trimestre. ${ }^{11}$ Particularmente a anemia por deficiência de ferro é definida pela hemoglobina menor que $11 \mathrm{~g} / \mathrm{dL}$ e ferritina menor que $12 \mu \mathrm{g} / \mathrm{L}$. Nas mulheres saudáveis, a dosagem de ferritina sérica é um marcador razoável de reserva de ferro que pode ser mobilizada, fornecendo com isso, o status de ferro. A concentração de ferritina abaixo de 15-20 $\mu \mathrm{g} / \mathrm{L}$ indica depleção e deficiência de ferro. Quando somado a isso existe diminuição da hemoglobina, o critério para anemia ferropriva é substancial. O melhor momento para detectar risco associado de anemia na gravidez é no primeiro trimestre antes da expansão do volume plasmático. As quantidades de glóbulos vermelhos e volume plasmático materno aumentam na gravidez, mas isso não ocorre de forma simultânea. Hemoglobina e hematócrito diminuem ao longo do primeiro e segundo trimestres, sendo seu limiar mais baixo no final do segundo trimestre e início do terceiro trimestre. As taxas começam a se elevar novamente próximo ao termo. $\mathrm{O}$ pico da hemodiluição ocorre entre 24 e 26 semanas. ${ }^{12}$

Os fatores de risco para a anemia ferropriva incluem: uma dieta deficiente em alimentos ricos em ferro (ostra, fígado, carne vermelha, camarão, cereais enriquecidos, feijão, linhaça); carente de facilitadores da absorção do ferro (suco de laranja e de limão, morango, brócolis, pimenta) e rica em alimentos que dificultam a absorção de ferro (soja, chocolate, café, chá); desordens gastrintestinais; hipermenorreia; pequeno intervalo interpartal e perda sanguínea após o parto normal.

As verminoses e a malária são também causas expressivas. Estima-se, por exemplo, que na ancilostomose, as perdas sanguíneas variem entre $2 \mathrm{ml}$ e 100 $\mathrm{ml}$, diariamente.

\section{Complicações na gravidez}

A anemia reduz a resistência da grávida a infecções, aumenta as taxas de hemorragias ante e pós-parto e de parto pré-termo e eleva o risco de mortalidade materna. Além disso, a deficiência de ferro e a anemia ferropriva que não são tratadas no terceiro trimestre levam a repetição do quadro no pós-parto..$^{13}$ Nessa fase, a deficiência de ferro e a anemia estão associadas à diminuição das habilidades físicas, à instabilidade emocional, ao estresse e à redução dos níveis cognitivos quando testados. ${ }^{14,15}$

O ferro é um elemento importante para o desenvolvimento do cérebro fetal e de habilidades cognitivas do recém-nascido. ${ }^{16} \mathrm{O}$ crescimento do cérebro demanda uma suplementação de ferro balanceada através da bar- reira hematoencefálica. ${ }^{17}$ No feto e no recém-nascido, a deficiência de ferro pode causar dano permanente ao cérebro e afetar de forma negativa a inteligência, as habilidades cognitivas e o comportamento durante a infância e a idade adulta. ${ }^{18}$ Crianças nascidas de mães que tiveram suplementação de ferro têm menor risco de desenvolver deficiência de ferro e anemia no primeiro ano de vida. ${ }^{19}$ Além disso, apresentam maior reserva de ferro (ferritina sérica) que crianças nascidas de mãe que usaram placebo. ${ }^{4,20}$

Outro fato importante que influencia no status de ferro do recém-nascido é o volume de sangue que é transferido da placenta antes do cordão umbilical ser clampeado. Em bebês a termo, a espera por pelo menos dois minutos após o nascimento para que o cordão umbilical seja clampeado é benéfica tanto para o status hematológico como para o status de ferro. ${ }^{21}$ Isso faz com que o volume sanguíneo do recém-nascido aumente em aproximadamente $30 \%$ e, com isso, a deficiência de ferro durante a infância diminua. ${ }^{22}$

Outros estudos mostram que o contrário também pode estar associado a resultados gestacionais adversos. A hemoglobina materna elevada foi associada com falência de expansão do volume plasmático e, com isso, relacionada a complicações como: hipertensão materna, pré-eclâmpsia e diabetes. ${ }^{23}$ Dessa forma, fornecer doses elevadas de ferro a mulheres sem anemia pode aumentar a hemoconcentração e provocar resultados adversos.

\section{Profilaxia e tratamento}

O Congresso Americano de Obstetras e Ginecologistas (ACOG, 2008) refere que uma dieta convencional contém $15 \mathrm{mg}$ de ferro elementar por dia. As necessidades de ferro elementar diárias na gravidez são de 27 $\mathrm{mg}$, quantidade geralmente presente nos suplementos vitamínicos. ${ }^{11}$ Por outro lado, a Organização Mundial da Saúde (OMS, 2008) recomenda na gravidez $60 \mathrm{mg}$ por dia de ferro elementar e o Institute of Medicine (IOM, 2001), $45 \mathrm{mg}$ por dia. ${ }^{3,24}$ As metanálises de estudos randomizados sobre o uso do ferro no período pré-natal, mostraram uma redução linear na anemia materna com doses maiores de ferro, acima de $66 \mathrm{mg}$ por dia. ${ }^{25}$

Nos países desenvolvidos, a profilaxia da anemia guiada pelo status de ferro da gestante, obtido pela ferritina sérica, tem vantagens fisiológicas sobre a profilaxia universal. Por outro lado, na maioria dos países em desenvolvimento, éindicada a profilaxia universal de ferro, e altas doses de ferro por via oral podem ser 


\section{Artigo de revisão}

recomendadas de acordo com o status de ferro em populações específicas de mulheres em idade fértil e gestantes. ${ }^{16}$ Não existe um consenso nos países ocidentais acerca do uso profilático de ferro na gestação. Alguns países como a Dinamarca preconizam a administração de ferro profilático enquanto outros como a Noruega não recomendam tal prática.E ainda há aqueles, como a Alemanha, que ainda não estabeleceram um protocolo padronizado. No Brasil, apesar de o Ministério da Saúde recomendar a suplementação de $40 \mathrm{mg}$ por dia de ferro para todas as gestantes com hemoglobina maior ou igual a $11 \mathrm{~g} / \mathrm{dL}$ a partir da $20^{\mathrm{a}}$ semana, alguns serviços seguem o protocolo de alguns países desenvolvidos na prescrição do ferro baseado no nível de hemoglobina da paciente. ${ }^{26}$ A OMS recomenda que a suplementação de ferro deva ser feita até 12 semanas após o parto nos países em desenvolvimento. ${ }^{27}$

Contudo, a suplementação dietética no pré-natal é o principal fator profilático, sendo relevante o uso de proteína animal e de vegetais ricos em ferro. O planejamento pré-natal também é importante, espaçando os intervalos interpartais para dois anos, ou até para três anos em pacientes malnutridas. Os anticoncepcionais orais merecem considerações especiais em anêmicas crônicas pela prevenção da gravidez e pela redução do sangramento menstrual.

Nas grávidas com anemia, o tratamento será feito com o sulfato ferroso, 200-400 mg, três vezes ao dia, via oral; excepcionalmente, administra-se o ferro pela via intravenosa (sacarato de hidróxido de ferro III). O tratamento das verminoses é igualmente importante. Quando a hemoglobina é menor que 6-7 g/dl, indica-se o concentrado de hemácias.

\section{Anemia macrocítica}

As anemias macrocíticas mais relevantes incluem aquelas por deficiência de folato (anemia megaloblástica) e de vitamina B12 (anemia perniciosa). A anemia macrocítica está caracterizada por VCM maior que 100 fl. O diagnóstico pode ser confirmado pela avaliação da concentração sérica de ácido fólico e de vitamina B12.

A anemia por deficiência de ácido fólico está associada à dieta pobre em vegetais folhosos frescos, legumes e proteínas animais. Na gravidez, as necessidades de ácido fólico diárias aumentam de 50 para $400 \mu \mathrm{g}$. O tratamento é feito com alimentos ricos em ácido fólico e suplementação oral, $1 \mathrm{mg}$ por dia.

A anemia macrocítica por deficiência de vitamina B12 (anemia perniciosa) pode ser encontrada em mulhe- res submetidas à gastrectomia total ou com doença de Crohn. Aquelas gastrectomizadas podem requerer 1.000 $\mu \mathrm{g}$ de vitamina B12 em intervalos mensais.

\section{Conclusão}

A anemia pode ser considerada um importante agravo na gestação, causa de importante morbimortalidade materna e fetal em alguns países. O diagnóstico da anemia bem como seu tratamento e profilaxia devem ser considerados em todo acompanhamento pré-natal. A gestação, por suas características fisiológicas próprias, pode predispor a mulher a desenvolver quadro anêmico, que pode ser agravado pelo curto intervalo gestacional, bem como pele carência nutricional. Os protocolos de prevenção e tratamento da anemia em diversos países são diferenciados e seguem padrões próprios de acordo com o perfil epidemiológico a ser abordado.

\section{Referências}

1. Khan KS, Wojdyla D, Say L, et al. WHO analysis of causes of maternal death: a systematic review. Lancet, 2006;367(9516):1066-74

2. Milman N. Anemia - still a major health problem in many parts of the world. Ann Hematol. 2011;90(4):369-77. http://dx.doi. org/10.1007/s00277-010-1144-5

3. World Health Organization. Worldwide prevalence of anemia report 1993-2005: WHO global database on anemia. Geneva: World Health Organization;2008. Acesso: 29/05/2014 Disponível em: http://whqlibdoc.who.int/publications/2008/9789241596657_eng.pdf

4. Milman N. Iron prophylaxis in pregnancy - general or individual and in which dose? Annhematol. 2006;85(12):821-8.

5. Olivares M, Pizarro M, Ruz M, et al. Acute inhibition of iron bioavailability by zinc: studies in humans. Biometals. 2012;25(4):657-64. http://dx.doi.org/10.1007/s10534-012-9524-z

6. Viteri FE, Casanueva E, Tolentino MC, et al. Antenatal iron supplements consumed daily produce oxidative stress in contrast to weekly supplementation in Mexican non-anemic women. Reprod Toxicol. 2012;34(1):125-32. http://dx.doi.org/10.1016/j. reprotox.2012.03.010

7. Milman N, Bergholt T, Eriksen L, et al. Iron prophylaxis during pregnancy - How much iron is needed? A randomized dose-response study of 20-80 mg ferrous iron daily in pregnant women. Acta Obstet Gynecol Scand. 2005;84(3):238-47

8. Milman N. Iron and pregnancy - a delicate balance. Ann Hematol. 2006;85(9):559-65

9. World Health Organization. Iron deficiency anaemia: assessment, prevention and control. WHO/NHD, 2001.

10. Bothwell TH. Iron requirements in pregnancy and strategies to meet them. Am J ClinNutr. 2000;72(1 Suppl):257S-264S

11. American College of Obstetricians and Gynecologists. Anemia in pregnancy. Practice Bulletin No 95. Obstet Gynecol. 2008;112(1):201-7. http://dx.doi.org/10.1097/ AOG.0b013e3181809c0d

12. Institute of Medicine. Nutrition during Pregnancy. Washington, 
Carlos Antonio B. Montenegro e cols. • Anemia e gravidez

DC: National Academy Press; 1990.

13. Milman, N. Postpartum anemia I: definition, prevalence, causes and consequences. Ann Hematol. 2011;90(11):1247-53. http:// dx.doi.org/10.1007/s00277-011-1279-z

14. Beard JL, Hendricks MK, Perez EM, et al. Maternal iron deficiency anemia affects postpartum emotions and cognition. J Nutr. 2005;135(2):267-72

15. Corwin EJ, Murray-Kolb LE, Beard JL. Low hemoglobin level is a risk factor for postpartum depression. J Nutr. 2003;133(12):4139-42

16. Milman N. Postpartum anemia II: prevention and treatment. Ann Hematol. 2012;91(2):143-54. http://dx.doi.org/10.1007/ s00277-011-1381-2

17. Beard J. Iron deficiency alters brain development and functioning. J Nutr. 2003;133(5 Suppl 1):1468S-72S

18. Grantham-Mc Gregor S, Ani C. A review of studies on the effect of iron deficiency on cognitive development in children. J Nutr. 2001;131(2S-2):649S-68S

19. Michaelsen KF, Milman N, Samuelson G. A longitudinal study of iron status in healthy Danish infants: effects of early iron status, growth velocity and dietary factors. Acta Paediatr. 1995;84(9):1035-44

20. Milman N, Agger AO, Nielsen OJ. Iron supplementation during pregnancy. Effect on iron status markers, serum erythropoietin and human placental lactogen. A placebo controlled study in
207 Danish women. Dan Med Bull. 1991;38(6):471-6

21. Hutton EK, Hassan ES. Late vs early clamping of the umbilical cord in full-term neonates: systematic review and meta-analysis of controlled trials. JAMA. 2007;297(11):1241-52

22. Moller NK, Weber T. Early vs delayed clamping of the umbilical cord in full term, preterm and very preterm infants. UgeskrLaeger. 2008;170(22):1919-21

23. Pena-Rosas JP, Viteri FE. Effects and safety of preventive oral iron or iron + folic acid supplementation for women during pregnancy. Cochrane Database Syst Rev. 2009;(4):CD004736. http://dx.doi.org/10.1002/14651858.CD004736

24. Institute of Medicine. Dietary Reference intakes for vitamin A, Vitamin K, Arsenic, Boron, Chromium, Copper, lodine, Iron, Manganese, Molybdenum, Nickel, Silicon, Vanadium, and Zinc. National Academy Press .Washington DC; 2001

25. Haider BA, Olofin I, Wang M, et al. Anemia, prenatal iron use, and risk of adverse pregnancy outcomes: systematic review and meta-analysis. BMJ 2013;346:f3443.

26. Ministério da saúde. Gestação de alto risco - Manual Técnico

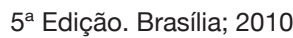

27. Stoltzfus R, Dreyfuss M. Guidelines for the use of iron supplements to prevent and treat iron deficiency anaemia. The International Nutritional Anaemia Consultative Group, (INACG/ WHO/UNICEF).Washington DC; 1998. 